\title{
The balance equation: Part 1. Response-specific inhibition and the operant-contingency puzzles
}

\author{
DOUGLAS ANGER \\ University of Missouri-Columbia, Columbia, Missouri
}

\begin{abstract}
This article and Part 2 (Anger, in press) present a new molecular analysis of operant reinforcement and extinction. In this multifactor analysis, response competition can influence extinction, but the present focus is on the influences of other basic extinction processes that can be isolated and studied with procedures that minimize the effects of competing behavior. Part 1 shows that one extinction process, response-specific inhibition (RSI; Anger, 1983), can account for animal sensitivity to complex response contingencies. RSI depends on the same events that determine whether a reinforcer is contingent on a response. RSI can average those event frequencies, combine them into a single variable that varies with the contingency, and adjust responding appropriately. Part 2 uses the findings of Part 1 to derive a rational equation that describes operant responding. This linear additive equation specifies a balance between RSI-augmenting events (e.g., unreinforced responses) and RSI-decrementing events (e.g., reinforced responses). The equation predicts a different relation between responding and reinforcer frequency than do other theories, because it predicts that responding is augmented by an unrecognized variable, unreinforced nonresponse time during controlling stimuli.
\end{abstract}

Evidence has been reported (Anger, 1983) that a responsespecific inhibition (RSI) controls the frequency of members of each class of operant responses. In the present pair of articles (see also Anger, in press), it will be shown that the concept of RSI together with other findings generate via rational steps a remarkably simple and novel equation for the description of operant behavior. This equation seems to describe a variety of complex relations found in operant behavior, and is quite different from other equations proposed for that purpose. The new analysis and equation (1) make unexpected, yet testable, predictions; (2) reveal the importance of a previously neglected behavior variable; and (3) produce a reasonable explanation for how animals can detect operant contingencies even though such detection requires averaging several different events over a substantial time period.

\section{RESPONSE-SPECIFIC INHIBITION}

Because the concept of RSI is basic to this analysis, a brief review is provided of this concept and the evidence for it. Examination of the full case (Anger, 1983) is recommended. Trials were given during which a small wheel was briefly introduced into a rat's cage. The rat's first turns on a trial were intermittently reinforced. After turning occurred on all trials, reinforcements were decreased until nonturns occurred

This research was funded in part by a grant from the Research Council of the Graduate School, University of Missouri-Columbia. This analysis was presented at the Association for Behavior Analysis meeting in Columbus, $\mathrm{OH}, 1985$. The author wishes to thank Kathleen Anger, Lynn Hammond, Eliot Hearst, Richard Herrnstein, Andrew Homer, Duncan Luce, Marjorie Marlin, William Timberlake, Trish Vandiver, Edward Wasserman, and Raymond Wolf for valuable comments. Reprints may be obtained from the author, Department of Psychology, McAlester Hall, University of Missouri, Columbia, MO 65211. on about $5 \%$ of the wheel introductions. Then some extra food pellets were selectively given about $6 \mathrm{sec}$ after the end of trials without any turns; hence, the pellets occurred about $10 \mathrm{sec}$ after the usual time of turn occurrence. Turns decreased. When the delayed reinforcements of nonturns were stopped, turns increased. Reinforcers have little reinforcing effect on a response when delayed until $10 \mathrm{sec}$ after it, if conditioned reinforcers are eliminated (Grice, 1948). Hence, the 10-sec delay of reinforcers after the nonturns should have minimized reinforcement of competing responses during the times when nonturning occurred. Consequently, the turn decrease did not seem to be due to reinforcement of competing responses.

The above evidence, together with other controls, indicated that during the nonturns, some event-a responseeliminating event-was occurring that could be reinforced as a response can be. However, that event was different from a response, since it had a different delay of reinforcement gradient; hence, the event did not seem to be competing behavior. The term behavioral inhibition appears appropriate for such an event having properties different from a competing response, yet preventing a response, and resulting from the stimulation of additional reinforcers. Response decreases due to added stimulation have been accepted as a basic characteristic of inhibition (Hearst, 1972, pp. 9-10). This kind of behavioral inhibition has been called responsespecific inhibition to distinguish it from conditioned inhibition, a less response-specific kind of inhibition (Hearst, 1972; Rescorla, 1969).

Operant reinforcement is viewed as having two effects, an initial effect that selects a class of responses and brings them under the control of a group of stimuli $(\mathrm{S}+)$. With continued reinforcement of one response class (hereafter called a response), the selective effect seems to stabilize early, and then the second effect, RSI, controls the frequency of that 
response. Each class of responses is controlled by a RSI specific to that class. The excitatory ability of the $S+s$ is determined by the frequency of reinforcers during them. However, the RSI of different responses is a major determinant of which responses occur during an S+. The RSI is a separate operant process controlled by the relative occurrence of reinforcers and other events following that response during the $\mathbf{S}+\mathrm{s}$. This article is primarily concerned with RSI and an equation for RSI that describes how the events following a response control the frequency of that response. However, the processes that control the excitatory ability of the $\mathbf{S}+$ will have to be considered when we examine the experimental testing of RSI predictions.

\section{MULTIFACTOR THEORY}

This analysis is part of a multifactor theory of extinction and schedule behavior. The S+ control (1) and RSI (2) have been mentioned, but during ordinary extinction there also seems to be a different generalized depression (3) of responding (Anger, 1983) that will be considered in another article (Anger, 1988). A particular response can be affected by unconditioned responses and other well-reinforced responses; hence, competing responses (4) can influence a response under study. The present viewpoint is that procedures can be found in which the response under study is not seriously influenced by other responses, but instead is controlled by RSI and the S+ control just mentioned. It is not denied that a response occurs in a context of other behavior. Rather it is proposed that contexts can be found where the contextual behaviors have sufficiently small effects on the response that the major actions of RSI and S+ control can be measured without serious error. Abrupt changes in major stimuli can produce generalization-decrement (5) effects. For example, with $100 \%$ reinforcement, no responses are reinforced after unreinforced responses, but then an abrupt reinforcement decrease results in many responses occurring after unreinforced responses, which provide new stimuli. The behavior may be affected by the lack of past reinforcement after the new stimuli. That generalization-decrement effect can be minimized by a slow reduction of reinforcement frequencies that never exposes the animal to frequencies of unreinforced responses very different from frequencies that have already preceded reinforcement. Thus it appears possible, though not easy, to minimize the effects of generalized inhibition, competing behavior, generalization decrement, and other factors, so that the major subjects of this paper-RSI and $\mathrm{S}+$ control-can be isolated and characterized.

\section{TERMS AND DEFINITIONS}

Let us use $P$ to represent a response, more specifically, the occurrence in a behavior sample of a member of a particular class of operant responses, such as pressing a bar or pecking a key. Let $P^{\prime}$ represent the absence of that response during the stimulus in whose presence the response is sometimes reinforced, hereafter called S+. According to the present analysis, when the other factors mentioned in the preceding paragraph are eliminated, then nonresponses, $P^{\prime}$ s, in the presence of $S+$ result from RSI (provided the animal is motivated, healthy, awake, etc.). Let $r$ represent an oc- currence of a reinforcer, such as food, and $r^{\prime}$ represent the absence of that reinforcer. Then $P, r$ will represent a response closely followed by a reinforcer; $P, r^{\prime}$ will represent a response not closely followed by the reinforcer (an unreinforced response); and $P^{\prime}, r$ a reinforcer that occurs after nonoccurrence of the response during an $\mathrm{S}+$ (i.e., $P^{\prime}, r$ represents a reinforcement of the RSI of that response). The term sequence seems the best available name for the group of above-defined sequences $P, r ; P, r^{\prime} ;$ and $P^{\prime}, r$. When these expressions for events and sequences are enclosed in brackets, they will refer to the rate of those events, the sequences per hour (or other time unit of the period studied), or the relative frequencies of those sequences in a particular behavior sample. Thus $\left[P, r^{\prime}\right]$ should be read as "the rate of unreinforced responses." (The commas within sequences distinguish the sequences from products.) The expressions without brackets will be used to refer to individual cases of the sequences. Thus $P, r^{\prime}$ is read as "an unreinforced response," and $P, r$ 's is read as "unreinforced responses." The term $\left[t_{s_{+}}\right]$will refer to the duration of $S+$ per time unit of the period studied.

\section{$\boldsymbol{P}^{\prime}, \boldsymbol{r}^{\prime}, \mathrm{S}+$ WITHOUT $\boldsymbol{P}$ OR $\boldsymbol{r}$}

The effects of $P, r, P, r^{\prime}$, and $P^{\prime}, r$ sequences on responding are well established. The empirical effect of $P^{\prime}, r$ has been well demonstrated by the results with DRO (differential reinforcement of other behavior) schedules (e.g., Uhl \& Garcia, 1969; Zeiler, 1971), although these results have been given a different theoretical interpretation. The present proposal is that all three sequences affect RSI, which in turn controls $[P]$ and $\left[P^{\prime}\right]$.

Clearly there is a fourth sequence, $P^{\prime}, r^{\prime}$, that needs consideration for completeness, but also because contingency often cannot be estimated without estimating $P^{\prime}, r^{\prime}$. The $P^{\prime}, r^{\prime}$ sequence occurs when $\mathrm{S}+$ occurs without either a $P$ or an $r$. Sometimes trials with the $P^{\prime}, r^{\prime}$ sequence can be counted as are $P, r$ and $P, r^{\prime}$, but often $P^{\prime}, r^{\prime}$ must be measured as a time duration, the time during $S+$ without $P$ or $r$. Sometimes that temporal duration will be emphasized by using $P^{\prime}, r^{\prime}$ time as equivalent to $P^{\prime}, r^{\prime}$. The temporal duration of such $P^{\prime}, r^{\prime}$ per time unit of the period studied will be represented as $\left[P^{\prime}, r^{\prime}\right]$.

The important behaviorial effects of $P, r, P, r^{\prime}$, and $P^{\prime}, r$ suggest that $P^{\prime}, r^{\prime}$ may affect behavior too. To be consistent with the effects of the other sequences, and to fit a role in detecting contingencies, $P^{\prime}, r^{\prime}$ should increase responding, since each negation reverses the effect. Thus double negation should have the same direction of effect as no negation, $P, r$. Since $P^{\prime}, r^{\prime}$ is due to RSI, it follows that the occurrence of RSI weakens the tendency for further RSI. Hence, when RSI occurs it requires augmenting sequences (such as $P, r^{\prime}$ or $P^{\prime}, r$ ) for its maintenance, or else it decreases. The name dissipation of inhibition, suggested by Anger (1983), seems appropriate for such an effect of $P^{\prime}, r^{\prime}$. Thus two sequences, $P, r^{\prime}$ and $P^{\prime}, r$, increase RSI, thereby decreasing $[P]$, and two sequences, $P, r$ and $P^{\prime}, r^{\prime}$, decrease RSI, thereby increasing $[P]$.

The view that an active inhibitory process is occurring during $P^{\prime}, r^{\prime}$ meshes well with the view that $P^{\prime}, r^{\prime}$ increases responding. It is reasonable for an active inhibitory process such as RSI to have some cost or limitation, some negative feedback from its occurrence that would restrain it. In con- 
trast, if $P^{\prime}, r^{\prime}$ is due to the absence of a response tendency, low stimulation, or such factors, it is difficult to see why the occurrence of $P^{\prime}, r^{\prime}$ should raise $P$.

It was stated that $\boldsymbol{P}^{\prime}$ s are due to RSI only during the S+ for $P$, so that $P^{\prime}, r^{\prime}$ only occurs during $S+$. Thus the difference between $\mathrm{S}+$ and $\mathrm{S}-$ cannot be due to RSI, but must have a different source, apparently a discrimination that results from the more frequent pairing of $r s$ with $S+$ than with other stimuli. Another implication is that when the frequency of $[r]$ during an $S+$ drops low enough, then $S+$ extinction occurs, which will reduce responding. The roles of excitation and inhibition or of Pavlovian conditioning in these effects are unclear; hence the terms discrimination and $e x-$ tinction will be used here to refer to the behavioral effects of pairing and of not pairing $r s$ with stimuli without further theoretical commitment.

\section{RESPONSE CONTINGENCY}

Anger (1983) pointed out that the above RSI formulation provides an answer to two perplexing questions in behavior research. Question 1: Is behavior determined by contiguity, individual response-reinforcer pairing; or is it determined by contingency, which depends on the relative frequency of several events in an extended sample of behavior (e.g., Church, 1969; Rescorla, 1967; Staddon \& Frank, 1975)? Question 2: How can animals adjust their responding to response-reinforcer contingencies in which some reinforcers occur at other times than after the response, as apparently they are able to do (Hammond, 1980)? Such a contingency is not a discrete event that can directly influence behavior such as each of the four sequences. To adjust behavior appropriately to such contingencies, both humans and animals have to somehow average the frequencies of several events over a substantial time interval, then combine those averages in a certain way, and adjust their behavior according to that combination.

The full extent of the contingency problem is beyond the scope of this analysis; here only a core problem will be analyzed, the case represented by the "contingency table" where there are four combinations of the presence or absence of either of two events. That common situation has the essence of the basic contingency detection problem, and is the place to start. Thus there will not be consideration of delayed contingencies where reinforcers may be contingent on a $P$ but follow it after a few seconds or after a variety of time intervals. There is some evidence that animals can also use their remarkable timing abilities to detect contingencies with consistent delays, even delays as long as a minute between two stimuli (e.g., Kamin, 1965). That seems a separable problem.

Without delayed reinforcers, we cannot treat continuous cases, such as a free-operant procedure where responses and reinforcers can occur with any temporal relation. Continuous cases have, in addition to $P, r$ and $P, r^{\prime}$, a continuum of different events with different time intervals between $P$ and $r$ ranging from zero up to many seconds. These different sequences have a variety of different effects depending on the time separation; consequently that case is considerably more complex both mathematically and experimentally. Some of the problems will be discussed in another article (Anger, 1988.
Consequently, the discontinuous trial case will be used both for the mathematical development of this analysis, and later for its initial experimental testing. That simplification clarifies the development of the balance equation, and increases the rigor of its testing by minimizing the number of different effects to be evaluated. If the equation is successful in the trial case, then it can be generalized to the free-operant case. The simplest trial procedure has brief trials that permit only one $P$ or no $P$, and only one $r$ or no $r$, and either $r$ or $\boldsymbol{r}^{\prime}$ can occur either following a $\boldsymbol{P}$ or a $\boldsymbol{P}^{\prime}$. Thus we have the same four sequences $\left(P, r ; P, r^{\prime} ; P^{\prime}, r ;\right.$ and $\left.P^{\prime}, r^{\prime}\right)$ of the $2 \times 2$ contingency table, and the same four defined in the last section. With that procedure, trials with $P^{\prime}, r^{\prime}$ can be counted, just as trials with $P, r ; P, r^{\prime}$; or $P^{\prime}, r$ are counted.

Although there are a variety of different measures of contingency, one widely used is the difference between the conditional probabilities (Church, 1969; Gibbon, Berryman, \& Thompson, 1974; Hammond \& Paynter, 1983; Jenkins \& Ward, 1965; Rescorla, 1967). With the just described procedure, $r$ would be said to be contingent on $P$ when the difference between $[P, r] /[P]$ and $\left[P^{\prime}, r\right] /\left[P^{\prime}\right]$ is significantly different from zero. If we substitute to obtain the difference in terms of the above four sequences, we obtain:

$$
\frac{[P, r]}{[P, r]+\left[P, r^{\prime}\right]}-\frac{\left[P^{\prime}, r\right]}{\left[P^{\prime}, r\right]+\left[P^{\prime}, r^{\prime}\right]} \text {. }
$$

That difference will be referred to as the Probability Difference (Hammond \& Paynter, 1983).

The Probability Difference 1 shows that the presence and degree of contingency depend on all four sequences only if neither $[P, r]$ nor $\left[P^{\prime}, r\right]$ are zero. If $\left[P^{\prime}, r\right]=0$, then the right side of the difference becomes 0 no matter what the value of $\left[P^{\prime}, r^{\prime}\right]$. Thus when reinforcers occur only after $P \mathrm{~s}$, and never after a $P^{\prime}$, a contingency is present no matter what $\left[P^{\prime}, r^{\prime}\right]$ is (so long as it is not also zero). However, the Probability Difference 1 also shows that if neither $\left[P^{\prime}, r\right]$ nor $[P, r]$ is zero, then variation in $\left[P^{\prime}, r^{\prime}\right]$ can change the right side of the difference from 1.0 to very low no matter what $\left[P^{\prime}, r\right]$ is. Hence, when neither $\left[P^{\prime}, r\right]$ nor $[P, r]$ is zero, then all four sequences have to be known to determine the presence and degree of contingency. Let us use the term complex contingencies to refer to such contingencies where neither $[P, r]$ nor $\left[P^{\prime}, r\right]$ are zero. Thus the appropriate adjustment of behavior to complex contingencies requires estimation of $\left[P^{\prime}, r^{\prime}\right]$ as well as the other three sequences.

We know animals are sensitive to $[P, r],\left[P, r^{\prime}\right]$, and $\left[P^{\prime}, r\right]$, and so to detect complex contingencies they need only be sensitive to one more, $\left[P^{\prime}, r^{\prime}\right]$. Anger (1983) pointed out that $[P, r],\left[P, r^{\prime}\right]$, and $\left[P^{\prime}, r\right]$ all seem to affect the RSI that controls the response frequency. Thus, it is a small additional step to propose that $\left[\boldsymbol{P}^{\prime}, \boldsymbol{r}^{\prime}\right]$ also affects RSI, and that the RSI of a particular response is a single variable that is increased or decreased by each of the four sequences in such a way that the RSI averages the frequencies of the four sequences, combines them into one variable that roughly assesses for the animal the sign and degree of contingency, and adjusts the response frequency appropriately.

Let us elaborate further the above answer to Question 2, which proposes that RSI plays a central role in the adjustment of responding to contingencies: 
1. The primary inputs into the animal are the four different sequences: $[P, r],\left[P, r^{\prime}\right],\left[P^{\prime}, r\right]$, and $\left[P^{\prime}, r^{\prime}\right]$. All four are the sorts of events that can directly affect animal behavior, and many studies have shown that behavior is affected by at least the first three of them. The results of Anger (1983) indicate that RSI is affected by the same three.

2 . The effects of three sequences are in the correct direction for contingency detection. RSI is increased by $\left[P, r^{\prime}\right]$ and $\left[P^{\prime}, r\right]$, and is decreased by $[P, r]$, so if $\left[P^{\prime}, r^{\prime}\right]$ also decreases RSI, then RSI will have at least a rough relation to the degree of contingency as estimated by the Probability Difference 1 . In more detail and for food reinforcement, when $[P, r]$ greatly exceeds $\left[P, r^{\prime}\right]$, and $\left[P^{\prime}, r\right]$ is zero, then RSI will be zero, $[P, r] /\left([P, r]+\left[P, r^{\prime}\right]\right)$ will be close to one, and $\left[P^{\prime}, r\right] /\left(\left[P^{\prime}, r\right]\right.$ $\left.+\left[P^{\prime}, r^{\prime}\right]\right)$ will be zero. Hence a high positive contingency will be present; $P$ s are highly effective in producing $r s$, and $P$ 's are not. Zero RSI of $P$ s produces high $[P]$, the appropriate behavior for that contingency. If $[P, r]$ is decreased, and/or $\left[P^{\prime}, r\right]$ is increased, RSI will develop at some point and then increase. The $[P, r]$ decrease will reduce $[P, r] /\left([P, r]+\left[P, r^{\prime}\right]\right)$, and/or the $\left[P^{\prime}, r\right]$ increase will augment $\left[P^{\prime}, r\right] /\left(\left[P^{\prime}, r\right]+\right.$ $\left.\left[P^{\prime}, r^{\prime}\right]\right)$, until at some point they become equal. Then $r s$ will be independent of $P$ s and no contingency will be present. At that point RSI will be substantial, which is appropriate for an ineffective response. As $\left[P, r^{\prime}\right]$ and $\left[P^{\prime}, r\right]$ increase further relative to $[P, r]$ and $\left[P^{\prime}, r^{\prime}\right]$, RSI will increase even more, and $\left[P^{\prime}, r\right] /\left(\left[P^{\prime}, r\right]+\left[P^{\prime}, r^{\prime}\right]\right)$ will exceed $[P, r] /\left([P, r]+\left[P, r^{\prime}\right]\right)$ more and more, giving an increasingly negative contingency. This increasing RSI will eventually produce zero $[P]$, which is appropriate for a negative contingency where a $P$ is not just ineffective but instead reduces $[r]$.

3. If the RSI is incremented by each $P, r^{\prime}$ and $P^{\prime}, r$ and decremented by each $P, r$ and each $P^{\prime}, r^{\prime}$, then RSI would average each of those effects over a time period, and would combine them into one variable, RSI, that would have a rough relation to the degree of contingency estimated by the probability difference.

4. The RSI would then produce a response frequency roughly appropriate to the degree of contingency.

From this point of view, the answer to Question 1 concerning the relative roles of individual response-reinforcer pairings and contingencies is that both play a role. However, for complex contingencies, four sequences play a role, not only the three well-known ones, and those sequences are the primary inputs into the animal. They determine RSI, which has a rough sensitivity to contingency so that responding is somewhat controlled by contingency too.

The relation between RSI and contingency was called "rough," because there are indications that RSI cannot be said to be proportional to the degree of contingency:

1. There is no one correct mathematical scale of contingency; instead, statisticians use several different measures (Gibbon, Berryman, \& Thompson, 1974). Hammond and Paynter (1983) say "correlation does not refer to a particular relationship between two variables; correlation refers to a class of related, but widely different relationships specified by different mathematical formulas" (p. 531, emphasis in original).

2. The occurrence of superstitious behavior is rather direct evidence that under some conditions RSI is low even though no contingency is present.

3. Anger (1983) reported evidence that a $P^{\prime}, r$ is more ef- fective across a delay than is a $P, r$ (Experiment 3), an asymmetry that suggests a bias in favor of RSI under some conditions. Other factors besides the degree of contingency, such as conserving energy, maximizing reinforcers, detecting changed conditions, and so forth, may influence the control of RSI by the four sequences. Although some sensitivity to response contingency would be expected to increase the survival of a species, other factors also affect survival, and therefore may have come to influence RSI. Consequently, RSI would not necessarily be expected to be proportional to any contingency measure used by statisticians. For a species, the weighting constants of the four inputs into RSI may have resulted during evolution from the greater reproductive fitness of animals with those constants as compared with the same animal in the same environment with different constants. Consequently, the constants may vary between species and may seldom if ever correspond closely to any particular statistical measure of contingency. In Part 2, it will be seen that one constant probably varies with the nature of the response, and another with the nature of the reinforcer.

\section{REFERENCES}

ANGER, D. (1983). Reinforcement of inhibition. Journal of the Experimental Analysis of Behavior, 39, 213-226.

ANGER, D. (in press). The balance equation: Part 2. Derivation of the balance equation for response-specific inhibition. Bulletin of the Psychonomic Society.

ANGER, D. (1988). The balance equation: Its testing and relations to other research. Manuscript in preparation.

Church, R. M. (1969). Response suppression. In B. A. Campbell \& R. M. Church (Eds.), Punishment and aversive behavior. New York: Appleton-Century-Crofts.

GibBon, J., Berryman, R., \& Thompson, R. L. (1974). Contingency spaces and measures in classical and instrumental conditioning. Joumal of the Experimental Analysis of Behavior, 21, 585-605.

GRICE, G. R. (1948). The relation of secondary reinforcement to delayed reward in visual discrimination learning. Journal of Experimental Psychology, 38, 1-16.

HAMmOND, L. J. (1980). The effect of contingency upon the appetitive conditioning of free operant behavior. Journal of the Experimental Analysis of Behavior, 34, 297-304.

Hammond, L. J., \& PAYNTER, W. E. (1983). Probabilistic contingency theories of animal conditioning: A critical analysis. Learning \& Motivation, 14, 527-550.

HeARST, E. (1972). Some persistent problems in the analysis of conditioned inhibition. In R. A. Boakes \& M. S. Halliday (Eds.), Inhibition and learning (pp. 5-39). New York: Academic Press.

JENKINS, H. M., \& WARD, W. C. (1965). Judgement of contingency between responses and outcomes. Psychological Monographs: General and Applied, 79(Whole No. 594), 1-17.

KAMIN, L. J. (1965). Temporal and intensity characteristics of the conditioned stimulus. In W. F. Prokasy (Ed.), Classical conditioning: A symposium (pp. 118-147). New York: Appleton-Century-Crofts. Rescorla, R. A. (1967). Pavlovian conditioning and its proper control procedures. Psychological Review, 74, 71-80.

Rescorla, R. A. (1969). Pavlovian conditioned inhibition. Psycholog ical Bulletin, 72, 77-94.

Staddon, J. E. R., \& Frank, J. A. (1975). The role of the peck-food contingency on fixed-interval schedules. Journal of the Experimental Analysis of Behavior, 23, 17-23.

UhL, C. N., \& GARCIA, E. E. (1969). Comparison of omission with extinction in response elimination in rats. Journal of Comparative \& Physiological Psychology, 69, 554-562.

ZEILER, M. D. (1971). Eliminating behavior with reinforcement. Journal of the Experimental Analysis of Behavior, 16, 401-405.

(Manuscript received for publication April 23, 1987.) 